\title{
Stochastic models of war alliances
}

\author{
WILLIAM J. HORVATH and CAXTON C. FOSTER \\ Mental Health Research Institute, The University of Michigan
}

\section{Introduction}

Lewis F. Richardson, the British physicist and meteorologist, devoted much of his life and scientific work to the study of the quantitative aspects of warfare. It was his firm belief that wars arise from measurable relations between nations and groups and that an understanding of these relationships would contribute more to the furtherance of peaceful coexistence than would conventional diplomacy. In a sense, his work was pioneering. The application of the methods of measurement and analysis of the physical scientist to the statistics of wars was unique. However, wars represent only a particular type of social phenomenon and the widening interest in the use of mathematical models in the social and psychological sciences suggests that Richardson's work should be viewed in the broader context of the mathematical revolution in social sciences.

Richardson himself was fully aware of the fact that his studies were just a beginning in this new approach but he hoped that in initiating them, he would lay the foundations on which others could build. His book, "The Statistics of Deadly Quarrels" contains a number of mathematical models relating to the occurrence and prosecution of wars. In particular he sought a mathematical description of the data on the number of nations which fought on the two sides of each war in the 120-year period ending in 1940 (Richardson, 1960, pp. 247-87). He was able to fit the observed distribution of the number of belligerents on each side only by invoking a number of rather special assumptions. The best mathematical model which he could formulate involved the following assumptions: disputes occur at random over the globe; each dispute interests exactly eight nations; the probability of war about a dispute between every pair formed from those nations is 0.35 ; this probability is constant for all nations involved, at all times.

Our approach to the problem will be somewhat different. We shall assume that the observed distribution is the outcome of a stationary stochastic process, i.e., an equilibrium distribution of group size which results from the random interaction between nations, with, however, a very specific mechanism for the formation and dissolution of war alliances. The distribution obtained from such a process will, of course, depend on the particular method by which a nation is assumed to join and leave the warring groups. Several different models are discussed in this paper but the one which best fits the observed data results in the Yule distribution, a particular skew distribution function which has been found to occur in a wide number of different 
social and psychological situations. Not only does this function fit the data well, but the underlying assumptions used in its derivation are very reasonable and much more general than those required by the Richardson model described above.

\section{The Concept of Aggregation for Aggression}

Richardson, in the course of his wideranging studies, analyzed some data on frequency of occurrence of bandit raids of a given size which took place in Manchoukuo in 1935. He found that the number of raids per unit interval of size decreased as the 2.3 power of the number of bandits in the group (Richardson, 1960, p. 116). A study of the membership of Chicago gangs in the middle 1920's also quoted in this book showed as identical inverse 2.3 power decrease of numbers of gangs with the size of membership (p. 119). On the basis of these two observations, Richardson conjectured that they represented some fairly general tendency concerning aggregation for aggression.

In a critique of Richardson's work, Rapoport pointed out that distribution functions whose asymptotic behavior leads to an inverse power function with exponent greater than 2 can be explained by a stochastic process previously used by Simon to derive the Yule distribution function (Rapoport, 1957; Simon, 1955). Furthermore, he suggested that if the slope in question is indeed symptomatic of "organization for aggression," the study of other types of organizations might reveal significant differences in the underlying social and psychological processes at work.

Since the inverse power function is a valid approximation to the Yule distribution only for large values of group size, it would be desirable to test this model in the lower range of group sizes. This is especially im- portant since a recent study by Coleman and James of the equilibrium size distribution of small groups gathered for peaceful purposes (shopping, play groups, and public gatherings at a swimming pool), produced a distribution function (the truncated Poisson) which, while identical in all six cases tested, was distinctly different from the Yule distribution (Coleman and James, 1961). If there is indeed a difference in the equilibrium size distribution of peaceful and warring groups, it is important to establish the nature of this difference and relate it to the underlying stochastic process. Since neither set of data-the Manchurian bandit groups nor the Chicago gangs-are accurate at the small group sizes, an adequate comparison cannot be carried out on that basis. However, it is possible to use Richardson's data on the distribution of numbers of nations in war alliance for such a study since each nation may be considered as an individual unit in the stochastic process. Under these circumstances, the same distribution function would apply to nations as to individuals in the formation of groups.

\section{The Size of War Alliances}

Richardson's basic data on war coalitions is shown below in Table 1. This displays the number of nations on each side in the 91 wars which ended in the period between 1820 and 1939 and were of magnitude

TABLE 1

Number of Nations on Each Side in Wars of Magnttude Greater Than 3.5 Ending BETWEEN 1820 AND 1939 A.D.

\begin{tabular}{ccrrrrr}
\hline \hline $\begin{array}{c}\text { Number of } \\
\text { Nations }\end{array}$ & 1 & 2 & 3 & 4 & 5 & 6 \\
\hline 1 & 42 & 24 & 5 & 5 & 2 & 1 \\
2 & & 3 & 2 & 1 & 1 & 0 \\
3 & & & 0 & 1 & 0 & 0 \\
\hline
\end{tabular}

nb: Beyond the bounds of this table there were 2 wars of 7 vs. 1,1 war of 9 vs. 1 , and 1 war of 15 vs. 5 . 
greater than 3.5 (more than 3,160 dead). Note that this table does not differentiate between the two sides, so that for unequal numbers of opponents only the total numbers of wars with that combination of opponents is shown. Thus there were 24 wars with 2 nations on one side and 1 on the other. Since side A and side B have no absolute significance, no attempt is made to differentiate between wars of 2 versus 1 and 1 versus 2 .

Since each war was fought by two coalitions of nations, this gives a total of 182 separate coalitions that took part in wars during this historical period. Any particular nation may have participated in more than one war but each coalition which was formed during the period will be regarded as a separate statistical event. The number of occurrences of coalitions with a particular number of nations may then be obtained by summing the figures in Table 1 . The results are shown in Table 2 below.

TABLE 2

Number of Nations in Each War Coalition SHOWN IN TABLE 1

\begin{tabular}{lrrrrrrrr}
\hline \hline No. of Nations & 1 & 2 & 3 & 4 & 5 & 6 & 7 & $8+$ \\
No. of Occurrences & 124 & 34 & 8 & 7 & 4 & 1 & 2 & 2 \\
Theoretical No. & 129 & 29 & 11 & 5 & 3 & 2 & 1 & 2 \\
(Yule Distribution) & & & & & & & & \\
\hline
\end{tabular}

Undemeath the observed number of coalitions of each size there is shown the number which would be obtained if the sample were determined from a Yule distribution. These numbers were obtained by fitting the data using the method described by Simon (1955). The Yule distribution is determined by a single parameter, $\propto$, which in this case is the number of groups (182) divided by the number of countries involved (308) and is equal to 0.59 . The number of coalitions with just one nation on a side is then given by $f^{*}(1)=\frac{182}{2-\alpha}$ and the num- ber of coalitions with $i$ nations on a side may then be determined from the recursion formula:

$$
f^{*}(i)=\frac{(1-\alpha)(i-1)}{1+(1-\alpha) i} f^{*}(i-1)
$$

A comparison of the two sets of numbers shows an extremely close fit with a $\chi^{2}$ of 4.5 , giving a probability of 0.7 that the observed deviations are due to chance.

Before discussing the significance of this finding, we should make a brief comment on the validity of treating each coalition of nations as an independent statistical event. It might be argued that the same group of nations repeatedly enters into wars and that the data might be biased by particular political combinations which occurred throughout this historical period. Richardson has actually studied this question by a detailed examination of the historical facts. His studies showed that during this 120 year period "new" belligerents entered into wars at a constant rate and that approximately one-third of the belligerents during that period were in this category at any one time (Richardson, 1960, p. 171). This constant flux of new belligerents was certainly a factor in making for new coalitions. However, there was a tendency for certain alliances to persist and it is important to determine whether this factor could have seriously modified the data. Instead of testing for all possible factors of this nature, a rather simple and more general test was performed to determine whether coalitions of any given size showed a preference for fighting coalitions of any other size.

In order to make this comparison, it was assumed that for the distribution of coalition sizes given in Table 2, each coalition could enter into a war with any other, independent of the size of the coalition involved. The calculation using this independence assumption could then be compared with the 
actual observed number of pairings of the different sized coalitions. For example, 124 of the 182 coalitions or 68 per cent consisted of just a single nation. Thus the probability of war with a single nation on each side is $0.68 \times 0.68=0.46$ and the number of such wars in a sample of 91 would be $91 \times 0.46$ $=42.2$.

Similarly, the percentage of two-nation coalitions is 19 per cent so that the number of 2- versus 1-nation wars should be $0.68 \times$ $0.19 \times 91=11.6$. And, since one would expect an equal number of 1-versus 2-nation wars, the combined total for this category is 23.2. In this way it was possible to construct a table for the expected number of wars for each combination of coalition sizes and compare the results with the observed data in Table 1. The numbers obtained in this way are shown in Table 3.

TABLE 3

Expected Number of Wars for Each Stze Coalrtion Assuming Statistical INDEPENDENCE

\begin{tabular}{ccrcccc}
\hline $\begin{array}{c}\text { Number of } \\
\text { Nations }\end{array}$ & 1 & \multicolumn{1}{c}{2} & 3 & 4 & 5 & 6 \\
\hline 1 & 42.2 & 23.2 & 5.4 & 5.0 & 2.7 & 0.7 \\
2 & & 3.2 & 1.5 & 1.3 & 0.8 & 0.2 \\
3 & & & 0.2 & 0.1 & 0.1 & 0 \\
\hline
\end{tabular}

Since the numbers are almost identical with that shown in Table 1, we can conclude that given the distribution of sizes of alliances in Table 2 there is no tendency for large groups to fight large groups or vice versa and that any sized coalition is equally likely to fight any other sized coalition.

\section{Mathematical Models of Group Formation}

\section{A. GENERAL}

The application of a stochastic model to explain the observed equilibrium size distribution in collections of small groups was first used by Coleman and James (1961). They treated the observed size distribution of "freely-forming" small groups in various public situations as the outcome of a probabilistic process by which individuals join and leave groups. The equilibrium distribution obtained from their model, the truncated Poisson, gave an extremely good fit to their data.

A subsequent paper by Harrison White pointed out that the same equilibrium distribution may be obtained for a range of assumptions about the underlying stochastic process and that one could resolve this ambiguity only by studying the variation of equilibrium distribution with changes in the independent parameter (White, 1962). This comment applies to any similar situation in which an attempt is made to relate an empirically determined equilibrium distribution to a particular stochastic process giving rise to such a distribution. However, rather than accepting this as a severe criticism of model making, one should regard it as a challenge to further study of the phenomena under consideration. For example, in the data cited by Coleman and James, a direct measurement of the joining and leaving rates for different sized groups and in different population densities would clarify the situation and eliminate the inapplicable models.

Since the size distribution of war alliances differs from that found by Coleman and James for peaceful social situations, we shall present first a derivation of the Yule distribution appropriate to the war alliance case and then make some general comments about the effect of varying the assumptions as to the making and breaking of alliances on the observed equilibrium distribution.

B. THE OPEN SYSTEM MODEL FOR THE YULE DISTRIBUTION

The derivation below follows that given by Simon except that we shall consider the stochastic process to be continuous in time 
(Simon, 1955). Let us assume that new nations are continually entering into conflict at a constant rate, $r$. Each new nation coming into a war will have a constant probability $\alpha$ of fighting alone and a probability $(1-\alpha)$ of joining an alliance. Furthermore, the chance of a nation joining an alliance of a given size is proportional to the number of nations already belonging to alliances of that size. Finally when an alliance breaks up, all bonds are broken and each former participant leaves the system although it may return again as a new uncommitted nation. After this process has gone on for some time, $N^{*}$ nations will have been involved in wars and a total of $A$ alliances will have been formed. We shall consider this as a system of $N^{*}$ nations which has reached an equilibrium distribution of alliances containing $n_{1}$ single-nation alliances, $n_{2}$ alliances of two nations, etc. The total number of alliances will then be equal to $A=\sum_{j=1}^{\infty} n_{j}$. We will then take a snapshot of this distribution over a short time interval so that the total number of nations, $N^{*}$, alliances, $A$, and alliances of a given size, $n_{j}$, are all held constant as new nations enter coalitions and old coalitions break up.

From our first assumption that each new nation will have a constant probability, $\alpha$, of forming a new alliance we get $A=\alpha N^{*}$. In order to calculate the net change in the number of alliances of size $i$ in a unit time, we have to consider three terms. The first term takes into account the fact that a portion of the $r(1-\alpha)$ new nations which form alliances during this unit time interval will join alliances of size $j-1$, converting them to alliances of size $j$. Since the fraction of the total number of nations con-

tained in groups of size $j-1$ is $\frac{(j-1) n_{j-1}}{N^{*}}$, and since the rate at which new nations join alliances of a given size is proportional to the number of nations already in alliances of that size, then $\frac{r(1-\alpha)(j-1) n_{j-1}}{N^{*}}$ alliances of size $j-1$ will be converted to alliances of size $j$ per unit time. Similarly $\frac{r(1-\alpha) j n_{j}}{N^{*}}$ alliances will be lost from this group since they will be converted into alliances of size $j+1$ during this time. Finally, if $q$ is the breakup rate of alliances, $q n_{j}$ alliances of size $j$ disappear during this time. Since in stochastic equilibrium the number of alliances of a given size will be constant, we may write this equation as:

$$
\begin{aligned}
& \frac{d n_{j}}{d t}=r(1-\alpha)(j-1) \frac{n_{j}-1}{N^{*}}- \\
& \quad r(1-\alpha) \frac{n_{j}}{N^{*}}-q n_{j}=0 .
\end{aligned}
$$

In addition to this equation, we must take into account the fact that in an open system in equilibrium, the number of nations entering the system must equal the number leaving it. Since $q n_{1}$ single-nation alliances disappear per unit time, $q n_{2}$ two-nation alliances, etc., then $q \sum_{j=1}^{\infty} j n_{j}=q N^{*}$ nations are removed from the system per unit time. This must equal the reentry rate, $r$, so that

$$
q N^{*}=r
$$

Substituting (2) in (1) we obtain:

$$
\begin{aligned}
& \qquad(1-\alpha)(j-1) n_{j-1}=n_{j}[j(1-\alpha)+1] \\
& \text { hence } \frac{n_{j}}{n_{j-1}}=\frac{(1-\alpha)(j-1)}{(1-\alpha) j+1} .
\end{aligned}
$$

To get the number of single-nation alliances we must set the first term in (1) equal to $\alpha r$. 


$$
\begin{aligned}
\text { thus } \frac{d n_{1}}{d t} & =0=\alpha r-(1-\alpha) r \frac{n_{1}}{N^{*}}-q n_{1} \\
\text { or } \alpha & =\frac{n_{1}}{N^{*}}(1-\alpha+1) \\
\text { hence } n_{1} & =\frac{\alpha N^{*}}{2-\alpha}=\frac{A}{2-\alpha}
\end{aligned}
$$

Equations (3) and (4) together determine the equilibrium distribution of alliances and this is the Yule distribution as derived by Simon (1955).

C. THE CLOSED SYSTEM MODEL FOR A YULE DISTRIBUTION

Coleman and James in their paper consider only closed systems of groups in which all of the individuals remain in the system (Coleman and James, 1961). Such systems are probably more valid for some of the social situations considered by them but are not very appropriate for a model of war alliances. In order to indicate the difference between the two types of systems, the equivalent model for a closed system will be considered briefly.

In a closed system we assume that $N$ nations are continually involved in wars and that they are formed into groups of $n_{1}$ isolates, $n_{2}$ alliances of two nations, $n_{3}$ of three, and, in general, $n_{j}$ alliances of $j$ nations each, for a total of $A$ alliances. The same rules for joining and breakup of alliances will apply as before. Isolates will join alliances proportional to the number of nations in alliances of that size and alliances will break up completely and all former members will join the pool of isolates. There will be a constant probability, $p$, that an isolate will enter into an alliance per unit time and a constant probability, $q$, that an alliance will rupture during that same period of time.

The derivation of the equilibrium distribution will not be repeated here but the recursion formula is again that for a Yule distribution except that the free parameter is different. The formula this time is:

$$
\frac{n_{j}}{n_{j-1}}=\frac{\beta(j-1)}{\beta j+1}
$$

where $\beta=1-\frac{n_{1}}{N}$.

This model was also tested against the data in Table 2 and gave a somewhat worse fit than the open system model. In this case $\chi^{2}$ was 8.9 and $P \cong 0.2$.

\section{OTHER STOCHASTIC MODELS FOR GROUP FORMATION}

The equilibrium size distribution of war alliances was shown to be a consequence of a stochastic process with particular rules for the formation and rupture of alliances. The assumptions made were that the probability of an isolate joining an alliance of a given size is proportional to the number of nations in alliances of that size and that rupture of an alliance resulted in a complete breakup into isolates. Modification of these rules would lead to a different type of equilibrium distribution function. For example, if the probability of an isolate joining an alliance were independent of the size of the alliance and if alliances were to shrink in size by individual members leaving with a constant probability proportional to the size of the alliance, the equilibrium distribution would be truncated Poisson (Coleman and James' "peaceful" groups). In fact, using the different combinations of these two assumptions would lead to the four different types of equilibrium distributions shown in Table 4 below.

Still other modes of joining and dissolution are possible. For example, White postulates a "conflict-oriented leaving process" wherein difficulties arise between pairs of members in an alliance (White, 1962). 
TABLE 4

Equilibrum Distrubutions for DIFFerent Assumptions of Formation and Dissolution OF GROUPS

\begin{tabular}{|c|c|c|}
\hline ution & $\begin{array}{l}\text { Independent } \\
\text { of No. of } \\
\text { Nations in } \\
\text { Alliance }\end{array}$ & $\begin{array}{l}\text { Proportional to } \\
\text { No. of Nations } \\
\text { in Alliance of } \\
\text { Given Size } \\
\end{array}$ \\
\hline & & Yule \\
\hline $\begin{array}{c}\text { Loss of } 1 \\
\text { proporti }\end{array}$ & $\begin{array}{l}\text { Trun } \\
\text { Pois }\end{array}$ & $\begin{array}{l}\text { Fisher's Log. } \\
\text { Series }\end{array}$ \\
\hline
\end{tabular}

Since in an alliance of size $j$ there are $\frac{j(j-1)}{2}$ pairs, the loss of a member due to this process would be proportional to $j(j-1)$. This sort of dissolution process combined with a proportional formation process also leads to a truncated Poisson distribution. Thus, knowledge of the equilibrium distribution does not uniquely determine the nature of the underlying stochastic process. This can only be done by direct observation of the parameters involved in the formation and dissolution process itself.

\section{Summary}

The distribution of the number of nations which fought on the two sides of each war during the period 1820-1939 A.D. has been computed as the outcome of a stochastic process for the formation and dissolution of war alliances. The observed data agree remarkably well with a Yule distribution which postulates that nations join alliances of a given size at a rate proportional to the total number of nations in alliances of that size and that alliances break up into their constituent members at a constant rate in- dependent of size. Thus Richardson was correct in his conjecture that there was a difference between peaceful groups and "aggregation for aggression." The difference, however, merely lies in the particular rules by which the individual members join and leave the groups. It should be noted that these rules for formation and dissolution are in accord with intuitively reasonable assumptions concerning the dynamics operating in "aggressive" and peaceful groups. We would expect that a member is not "free" to leave the former as he is free to leave the latter. One does not just resign from a gang or war alliance. The Yule model reflects this behavior by assuming the complete dissolution of the group whenever a member leaves it. This does not mean, of course, that the defection of a single member destroys the group. "Cause" and "effect" in a model of this sort can be freely interchanged so that it can be more naturally interpreted as: no member leaves an aggressive group until the whole group is dissolved.

\section{REFERENCES}

Colmanan, J. S. and James, J. "The Equilibrium Size Distribution of Freely-Forming Groups," Sociometry, 24 (1961), 36-45.

RAPOPORT, ANATOL. "Lewis F. Richardson's Mathematical Theory of War," The Journal of Conflict Resolution, 1 (1957), 249-99.

Richardson, Lewis F. Statistics of Deadly Quarrels. Pittsburgh: Boxwood Press, 1960.

Smon, H. A. "On a Class of Skew Distribution Functions," Biometrika, 42 (1955), 42540.

Whrre, H. "Chance Models of Systems of Casual Groups," Sociometry, 25 (1962), 153-72. 